\title{
Letter to the editor: Potential causes of the decreased effectiveness of the influenza A(H1N1)pdm09 strain in live attenuated influenza vaccines
}

CS Ambrose ${ }^{1}$, H Bright ${ }^{2}$, R Mallory ${ }^{1}$

1. Medlmmune, Gaithersburg, MD, United States

2. Medlmmune, Speke, United Kingdom

Correspondence: Christopher S. Ambrose (ambrosec@medimmune.com)

Citation style for this article:

Ambrose CS, Bright H, Mallory R. Letter to the editor: Potential causes of the decreased effectiveness of the influenza A(H1N1)pdmog strain in live attenuated influenza vaccines. Euro Surveill. 2016;21(45):pii=30394. DOI: http://dx.doi.org/10.2807/1560-7917.ES.2016.21.45.303942

Article submitted on 03 November 2016 / accepted on 10 November 2016 / published on 10 November 2016

To the editor: We greatly appreciate the editorial by Penttinen and Friede summarising the data regarding recent observations in the United States (US) of decreased effectiveness of the influenza $A\left(\mathrm{H}_{1} \mathrm{~N}_{1}\right)$ pdmo9 strains (A/California/7/2009 and A/Bolivia/559/2013) included in live attenuated influenza vaccines (LAIV) [1]. Multiple hypotheses have been suggested as potential explanations for the reduced effectiveness compared with inactivated influenza vaccines (IIV). The most frequently cited hypotheses include poor replicative fitness of the $A\left(\mathrm{H}_{1} \mathrm{~N}_{1}\right)$ pdmog LAIV strains, vaccine-virus interference in the quadrivalent formulation, reduced LAIV replication due to preexisting anti-influenza immunity from prior influenza vaccinations, and poor thermostability of $A\left(\mathrm{H}_{1} \mathrm{~N}_{1}\right)$ pdmog LAIV strains. We have systematically evaluated each of these hypotheses and would like to share our assessments in case they might benefit ongoing international scientific discussions regarding LAIV effectiveness.

Based on evidence presently available to us, we believe that reduced replicative fitness of the $A / C$ alifornia and A/Bolivia $\left(\mathrm{H}_{1} \mathrm{~N}_{1}\right)$ pdmog LAIV strains is the most probable root cause for the reduced vaccine effectiveness (VE). From 2010/11 through 2013/14, LAIV VE in children aged 2-17 years against matched $A\left(\mathrm{H}_{3} \mathrm{~N}_{2}\right)$ and $B$ strains has been comparable to that observed with IIV [2,3]. In 2014/15, LAIV4 VE against mismatched $\mathrm{A}\left(\mathrm{H}_{3} \mathrm{~N}_{2}\right)$ strains was low, similar to that observed with IIV [2], and similar to that of $\operatorname{LAIV}_{3}$ against mismatched $\mathrm{A}\left(\mathrm{H}_{3} \mathrm{~N}_{2}\right)$ strains that are $\geq 8$-fold different by haemagglutination-inhibition assay $[4,5]$. Laboratory studies that we have conducted since April 2016 show that A/ California and $\mathrm{A} /$ Bolivia strains have reduced replication in a human alveolar cell line and in primary human nasal epithelium air-liquid cultures, as well as reduced binding to a2,6-linked sialic acid receptors-the primary receptor for influenza viruses in the human upper respiratory tract. Consequently, we are actively working to identify a new $\mathrm{A}\left(\mathrm{H}_{1} \mathrm{~N}_{1}\right)$ pdmog LAIV strain with replicative fitness superior to that of $A / C$ alifornia and A/Bolivia and similar to the replicative fitness of LAIV strains that previously demonstrated high levels of effectiveness in children.

In the context of reduced replicative fitness, vaccine-virus interference may have contributed to the observed reduced VE. However, vaccine-virus interference specific to the quadrivalent formulation appears to be an unlikely root cause of the reduced VE with LAIV. LAIV 3 demonstrated reduced VE against $A\left(\mathrm{H}_{1} \mathrm{~N}_{1}\right)$ pdmog in 2010/11 in the US [2] and 2012/13 in Germany [6]. Additionally, no VE was observed against $A\left(\mathrm{H}_{1} \mathrm{~N}_{1}\right)$ pdmog strains in a randomised placebo-controlled study in children aged 2-5 years with trivalent $A$ / Leningrad LAIV [7]. As reduced VE against $A\left(\mathrm{H}_{1} \mathrm{~N}_{1}\right)$ pdmog strains was observed with trivalent LAIV formulations, any effects of vaccine-virus interference do not appear specific to the quadrivalent LAIV formulation.

Because rates of vaccine coverage in the US have historically been higher compared with European countries, questions have been raised regarding the role of prior vaccination in the reduced $A\left(\mathrm{H}_{1} \mathrm{~N}_{1}\right)$ pdmog effectiveness [1]. Available data suggest that preexisting anti-influenza immunity due to prior vaccination is an unlikely root cause of the reduced VE observed with LAIV. In 2013/14 and 2015/16, the effect of prior season influenza vaccination on LAIV VE was evaluated in the US-based Centers for Disease Control and Prevention Flu VE and Influenza Clinical Investigation for Children (ICICLE) studies [8-10]. No statistically significant effect of prior season vaccination on LAIV VE was observed in either study in any season. Additionally, in the ICICLE study and in a large cohort study of children aged 24-35 months in Finland, most LAIV recipients were previously vaccinated. VE estimates trended higher among children vaccinated against influenza compared with unvaccinated children in the prior season in the ICICLE 2013/14 study (19\% (95\% confidence interval 
(CI): -80 to 64$)$ vs $9 \%(95 \% \mathrm{Cl}:-161$ to 68$))$; the ICICLE 2015/16 study (60\% (95\% Cl: 1 to 84$)$ vs 35\% (95\% Cl: -206 to 86$)$ ), and the Finland study ( $74 \%$ ( $95 \% \mathrm{Cl}: 48$ to $87)$ vs $25 \%(95 \% \mathrm{Cl}:-27$ to 56$)$ ) [11].

In 2013/14, with LAIV4 containing the A/California strain, a statistically significant correlation was observed between reduced LAIV VE against $\left(\mathrm{H}_{1} \mathrm{~N}_{1}\right)$ pdmog viruses and higher outdoor temperatures during LAIV lot unloading at US distributors [12]. In laboratory experiments, A/California demonstrated increased heat degradation [13], including experiments that simulated heat exposures that may have occurred during US distribution ( $33^{\circ} \mathrm{C}$ for 4 hours). Environmental heat exposure has also been suggested as a contributing factor to the lack of LAIV VE against $A\left(\mathrm{H}_{1} \mathrm{~N}_{1}\right)$ pdmog viruses in a randomised placebo-controlled study in children aged $<5$ years with trivalent $A$ /Leningrad LAIV [14]. However, reduced VE was also observed in 2015/16 with A/Bolivia, the strain chosen to replace A/California based on its being more heat stable [1]. Consequently, although the reduced thermostability of $A$ /California appears to have contributed to the low VE observed in 2013/14 in the US, it cannot explain the observations of reduced VE against $A\left(\mathrm{H}_{1} \mathrm{~N}_{1}\right)$ pdmog strains in 2015/16 as A/Bolivia was thermostable.

We have initiated a multifaceted scientific investigation into the causes of the recently observed reduced effectiveness of LAIV, with the goal of identifying a more effective $A\left(\mathrm{H}_{1} \mathrm{~N}_{1}\right)$ pdmog LAIV strain for potential inclusion in the 2017/18 LAIV formulation. All potential hypotheses continue to be evaluated. We welcome the input and support of the multiple stakeholders involved, including national public health agencies, the World Health Organization, and additional external scientific experts, as we work together to ensure that VE of LAIV is improved in future influenza seasons

\section{Conflict of interest}

Christopher S. Ambrose, Helen Bright, and Raburn Mallory are full-time employees of Medlmmune.

\section{Authors' contributions}

All authors participated in the drafting of this letter, reviewed it critically for important intellectual content, and approved the final version. years of age in $2013-2014$ in the United States. Vaccine. 2016;34(1):77-82. DOI: 10.1016/j.vaccine.2015.11.010 PMID: 26589519

4. Lum LC, Borja-Tabora CF, Breiman RF, Vesikari T, Sablan BP, Chay $\mathrm{OM}$, et al. Influenza vaccine concurrently administered with a combination measles, mumps, and rubella vaccine to young children. Vaccine. 2010;28(6):1566-74. DOI: 10.1016/j. vaccine.2009.11.054 PMID: 20003918

5. Klick B, Durrani S, Chan KH, Ip DK, Chou ES, Kwok HK, et al. Live attenuated seasonal and pandemic influenza vaccine in school-age children: a randomized controlled trial. Vaccine. 2013;31(15):1937-43. DOI: 10.1016/j.vaccine.2013.02.017 PMID: 23434387

6. Helmeke C, Gräfe L, Irmscher HM, Gottschalk C, Karagiannis I, Oppermann $\mathrm{H}$. Effectiveness of the 2012/13 trivalent live and inactivated influenza vaccines in children and adolescents in Saxony-Anhalt, Germany: a test-negative case-control study.PLoS One. 2015;10(4):e0122910. DOI: 10.1371/journal. pone.0122910 PMID: 25885063

7. Victor JC, Lewis KD, Diallo A, Niang MN, Diarra B, Dia $\mathrm{N}$, et al. Efficacy of a Russian-backbone live attenuated influenza vaccine among children in Senegal: a randomised, double-blind, placebo-controlled trial. Lancet Glob Health. 2016;S2214-109X(16)30201-7.PMID: 27746224

8. Centers for Disease Control and Prevention (CDC). Advisory Committee on Immunization Practices (ACIP) Summary Report. Meeting held: October 29-30, 2014; Atlanta, GA: CDC; 2014. Available from: http://www.cdc.gov/vaccines/acip/meetings/ downloads/min-archive/min-2014-10.pdf

9. Flannery B. LAIV vs IIV effectiveness: Summary of evidence since 2009. Presented at: Centers for Disease Control and Prevention Advisory Committee on Immunization Practices (ACIP) Meeting; 2016 Jun 22-23; Atlanta, GA. Available from: http://www.cdc.gov/vaccines/acip/meetings/downloads/ slides-2016-06/influenza-07-flannery.pdf

10. Caspard H, Belongia EA, Bernatoniene J, Clipper L, Congeni $B$, Faust S, et al. Multicenter study of the effectiveness of live attenuated influenza vaccine and inactivated influenza vaccine in children in 2015-2016 in the United States. Poster presented at: Options for Influenza Meeting; 2016 Aug 24-28; Chicago, IL.

11. Nohynek H, Baum U, Syriänen R, Ikonen N, Sundman J, Jokinen J. Effectiveness of the live attenuated and the inactivated influenza vaccine in two-year-olds - a nationwide cohort study Finland, influenza season 2015/16. Euro Surveill. 2016;21(38):30346. DOI: 10.2807/1560-7917. ES.2016.21.38.30346 PMID: 27684447

12. Caspard H, Coelingh KL, Mallory RM, Ambrose CS. Association of vaccine handling conditions with effectiveness of live attenuated influenza vaccine against $\mathrm{H}_{1} \mathrm{~N}_{1} p d m 09$ viruses in the United States.Vaccine. 2016;34(42):5066-72. DOI: 10.1016/j. vaccine.2016.08.079 PMID: 27613072

13. Cotter $\mathrm{CR}$, Jin $\mathrm{H}$, Chen $\mathrm{Z}$. A single amino acid in the stalk region of the $\mathrm{H}_{1} \mathrm{~N} 1 \mathrm{pdm}$ influenza virus HA protein affects viral fusion, stability and infectivity.PLoS Pathog. 2014;10(1):e1003831. DOI: 10.1371/journal.ppat.1003831 PMID: 24391498

14. Isakova-Sivak I. Use of live attenuated influenza vaccines in young children in resource-poor settings.Lancet Glob Health. 2016;S2214-109X(16)30247-9.PMID: 27746227

\section{References}

1. Penttinen PM, Friede MH. Decreased effectiveness of the influenza $\mathrm{A}\left(\mathrm{H}_{1} \mathrm{~N}_{1}\right)$ pdmog strain in live attenuated influenza vaccines: an observational bias or a technical challenge?Euro Surveill. 2016;21(38):30350. DOI: 10.2807/1560-7917. ES.2016.21.38.30350 PMID: 27684999

2. Chung JR, Flannery B, Thompson MG, Gaglani M, Jackson ML, Monto AS, et al. Seasonal effectiveness of live attenuated and inactivated influenza vaccine. Pediatrics. 2016;137(2):e20153279. DOI: 10.1542/peds.2015-3279 PMID: 26738884

3. Caspard H, Gaglani M, Clipper L, Belongia EA, McLean HQ, Griffin MR, et al. Effectiveness of live attenuated influenza vaccine and inactivated influenza vaccine in children 2-17 